\title{
LA GOBERNANZA LOCAL DEL TURISMO RURAL COMO RESPUESTA A LOS EFECTOS DE LA COVID-19
}

\author{
THE LOCAL GOVERNANCE OF RURAL TOURISM AS A RESPONSE TO \\ THE EFFECTS OF COVID-19 \\ Ángel Iglesias Alonso \\ Universidad Rey Juan Carlos. Madrid/España. \\ angel.iglesias@urjc.es \\ https://orcid.org/0000-0002-1951-0460
}

Recibido/Received: 11/05/2021

Modificado/Modified: 24/09/2021

Aceptado/Accepted: 13/12/2021

\section{RESUMEN}

Dentro de la industria turística, el turismo rural es un fenómeno innovador que contribuye a la revitalización y generación de desarrollo económico y cohesión social de muchos municipios en riesgo de involución demográfica. En razón de las restricciones a la movilidad y a las medidas de distanciamiento social que ha supuesto la crisis sanitaria de la COVID-19, es, sin duda, un sector que se ha visto gravemente afectado.

Pero, por otro lado, en el contexto de la amenaza a la salud que supone la pandemia de la COVID-19, y mientras esta amenaza sea percibida, es previsible que en el próximo futuro, los ciudadanos opten por el turismo rural como la opción de menor riesgo para sus destinos de ocio y descanso, de manera que el sector se encuentra ante nuevos retos y oportunidades derivados de este sobrevenido nuevo escenario.

En este contexto, nuestra tesis es que para hacer frente a esos nuevos retos el sector necesita adoptar modelos innovadores de gobernanza, de carácter colaborativo y que tengan en cuenta las especificidades de cada destino en particular y, para ello, se fortalezca la autonomía de los gobiernos locales para el diseño e implantación de políticas públicas de desarrollo turístico más adecuadas al lugar.

Por tanto, desde el marco analítico que provee el concepto de gobernanza local, y a partir de un estudio cualitativo y documental, analizaremos redes creadas "ad hoc" de actores públicos, privados y del tercer sector a raíz de la emergencia sanitaria. Nos ocuparemos de los motivos de la creación de redes, de sus impactos en las ya existentes, de sus principales impulsores y de su liderazgo, de las innovaciones introducidas, y de los retos derivados de la coordinación y gestión entre actores públicos y privados de las nuevas demandas exigidas al sector del turismo rural y de su integración con otras políticas de desarrollo local.

Con todo, este trabajo tiene un doble objetivo. Por una parte, contribuir a la generación de teoría acerca de la gobernanza local en relación con de la gestión turística y como instrumento de cohesión social y territorial y, en segundo lugar, proponer pautas de actuación para la mejora del sector turístico rural dirigidas a los responsables en la toma de decisiones relacionadas con el desarrollo local a través del fomento del turismo rural, en línea con las prioridades de cohesión social y territorial de la UE en el marco del Instrumento europeo de Recuperación (Next Generation EU).

\section{PALABRAS CLAVE}

Gobernanza local, turismo rural, COVID-19, Unión Europea, España 


\title{
SUMARIO
}

1. Introducción. 2. La gobernanza como marco de esta investigación. 3. Un apunte metodológico. 4. La gobernanza local durante la pandemia. 5. La gobernanza post-pandemia: problemas y retos. 6 . Conclusiones y limitaciones de este estudio. 7. Bibliografía

\begin{abstract}
Within the tourism industry, rural tourism is an innovative phenomenon that contributes to the revitalization and generation of economic development and social cohesion in many municipalities at risk of demographic involution. Due to the restrictions on mobility and the measures of social distancing that have resulted from the health crisis of COVID-19, it is undoubtedly a sector that has been seriously affected.

However, on the other hand, in the context of the health threat posed by the COVID-19 pandemic, and as long as this threat is perceived, it is foreseeable that in the near future, citizens will opt for rural tourism as the least risky option for their leisure and rest destinations, so that the sector faces new challenges and opportunities arising from this new scenario.

In this context, our thesis is that in order to face these new challenges, the sector needs to adopt innovative models of governance, of a collaborative nature and which take into account the specificities of each particular destination. To this end, the autonomy of local governments in the design and implementation of public policies for tourism development that are more appropriate to the place must be strengthened. Therefore, from the analytical framework provided by the concept of local governance, and based on a qualitative study, we will analyse networks created "ad hoc" of public, private and third sector actors as a result of the health emergency. We will deal with the reasons for the creation of networks, their impact on existing ones, their main drivers and leadership, the innovations introduced, and the challenges derived from the coordination and management between public and private actors of the new demands made on the rural tourism sector and its integration with other local national and European development policies (Next Generation EU).
\end{abstract}

\section{KEYWORDS}

Local governance, rural tourism, COVID-19, European Union, Spain.

\section{CONTENTS}

1. Introduction. 2. Governance as framework for this research. 3. A methodological note. 4. Local governance during the pandemic. 5. Post-pandemic governance: problems and challenges. 6 . Conclusions and limitations of this study. 7. References

\section{INTRODUCCIÓN}

La COVID-19 ha desencadenado una de las principales crisis que afectan profundamente a la economía mundial, en particular al mercado turístico mundial. Las actividades relacionadas con el turismo abarcan las interacciones entre organizaciones de diferentes industrias, personas y eventos en diversos subsistemas de la industria del turismo (Scott \& Laws, 2008). Por ello, el turismo es una de las industrias más afectadas por situaciones catastróficas por su extrema sensibilidad a las mismas (Jiang, Richtie y Beckendorf, 2019) $\mathrm{y}$, en tal sentido, la recuperación obliga a la colaboración y a la coordinación de acciones entre actores públicos y privados. Colaboración que, en el desarrollo del turismo rural ha sido puesta de manifiesto a través de la importancia de los grupos de acción local en torno al programa LEADER (Tirado y Hernandez, 2018; Panik, 2015).

En el caso del turismo rural la crisis se acentúa dada la vulnerabilidad de los municipios pequeños. Va de suyo que se trata de una crisis multifacética, de carácter desconocido tanto 
en sus orígenes como en sus efectos, por lo que su afrontamiento requiere la colaboración de diferentes actores que actúen de manera coordinada. Hasta ahora, existen algunos estudios que se han ocupado de cómo gestionar situaciones de crisis que afectan al turismo, pero muy pocas ocasionadas por catástrofes de salud pública (Novelli et al, 2018). En materia de gestión de la crisis, prácticamente todos los gobiernos han implantado medidas extraordinarias para hacer frente a la pandemia (Bouckaert et. al. 2020), pero los impactos de la misma tendrán que ser evaluados en todas sus dimensiones. En lo que se refiere al turismo rural una primera constatación es que, primero, el confinamiento generalizado seguido de otros parciales y, después, los cierres perimetrales han producido, en diversos grados una inmovilización de las personas, siendo la movilidad una de las premisas para la misma existencia de la actividad turística. Sin duda, las restricciones han provocado en el sector turístico una reducción de los ingresos, un incremento del desempleo y una infrautilización de equipamientos en magnitudes hasta ahora desconocidas.

En este contexto, el objetivo de este trabajo es identificar los primeros impactos en el turismo rural derivados de la crisis global originada por la COVID-19, así como las medidas tomadas para su afrontamiento. Sin duda, la comprensión de los efectos de una crisis global en el turismo rural debe abordarse desde perspectivas multidisciplinares, pero aquí acotaremos nuestro estudio desde la perspectiva de la gobernanza local y focalizado al caso de España. No obstante, este estudio de caso tiene también una vocación comparativa, por lo que nuestro propósito añadido es que sirva para ulteriores estudios comparados de carácter internacional. Así mismo, nuestra primera aproximación será de carácter cualitativo con la intencionalidad de que estas primeras indagaciones sirvan de base exploratoria que nos permita realizar ulteriores estudios con aproximaciones cuantitativas para su tratamiento estadístico. Para este primer análisis se han mantenido entrevistas semiestructuradas con responsables de la política y de la administración local, así como con representantes de asociaciones regionales de turismo rural; los resultados de estas entrevistas se han triangulado con documentos elaborados por instituciones públicas y privadas relacionadas con el turismo rural. No se nos oculta que la pandemia y sus consecuencias siguen vigentes, lo que hace difícil estudiar un fenómeno cuando aún se está desarrollando en todos sus aspectos. De la misma manera, en la fase actual de la pandemia es necesario poner de manifiesto la dificultad de realizar un estudio comprensivo acotado al sector del turismo rural en razón de las numerosas interrelaciones que mantiene con muchos otros.

Por consiguiente, aunque sea difícil delimitar un concepto unívoco de turismo rural debido a las múltiples manifestaciones que presenta (Sharpley y Roberts, 2004), conviene subrayar que es una actividad que no sólo contribuye al crecimiento económico, sino a la mejora de la calidad de vida en el ámbito rural (Petrovic et al, 2018; Martínez, 2019, entre otros). En esta medida, también están documentados los beneficios que para la salud física y psíquica aportan las actividades de turismo rural para aquellos que las realizan (Sharpley y Roberts, 2004; Sims, 2009; Kumar, 2020). Esta percepción de los beneficios que aporta el turismo rural para la salud puede ser un elemento incentivador de la actividad turística rural una vez esté superada la pandemia.

En cualquier caso, lo que es incuestionable es que la crisis desatada por la COVID-19 está teniendo importantes impactos sanitarios, sociales, económicos y también institucionales. La pandemia es, a corto plazo, un fenómeno que ha provocado una devastación en la salud pública y en la economía y, en su conjunto, una crisis multidimensional se pone de manifiesto con toda su crudeza en los pueblos y ciudades. Los efectos indeseados del virus se han manifestado en los municipios, sometiendo a las administraciones locales a una tensión desconocida $\mathrm{y}$, por tanto, como instituciones, los 
gobiernos locales, como administración más próxima al ciudadano, también se han visto afectados y abocados a adoptar estrategias de respuesta y, con ello, la crisis de la COVID19 ha influenciado las relaciones de gobernanza en aspectos que no sólo tienen que ver con las interacciones entre los gobiernos locales y otros gobiernos territoriales, central y regional, sino también con la sociedad civil. Todo ello en un contexto donde, primero, ni el gobierno local ni la gobernanza local en España está entre las prioridades de los partidos políticos y en una situación donde los gobiernos locales, afectados por las políticas de austeridad derivados de la crisis económica de 2008, se han visto obligados a enfrentarse a una crisis extra-sistémica. Así las cosas, los gobiernos se enfrentan cada vez más a problemas diabólicos (wicked problems) para los que no existen a priori soluciones previas y estas soluciones están abocadas a confrontar innumerables conflictos de valores, objetivos y para dar solución los gobiernos dependen de redes. La COVID-19 refuerza esa tendencia. En primer término, la urgencia de la pandemia ha exigido reforzar la coordinación y colaboración con los otros niveles territoriales. A esta diversidad se une la diferente incidencia de la crisis extra-sistémica en función del territorio. Lo que es innegable es que las desigualdades se manifiestan en los territorios concretos y los gobiernos locales tienen que actuar para hacer frente a esos problemas. Sin duda, aunque el sector público está abocado a liderar la recuperación, en el proceso las pequeñas y medianas empresas tienen un papel principal y, en el caso de las empresas de turismo rural, estas están llamadas a desempeñar un papel protagonista en la recuperación de los núcleos rurales en los que operan (Foris, 2014).

Como ha quedado señalado, el objetivo del presente trabajo es contribuir a la investigación que se está desarrollando sobre el fenómeno, pero también aportar insumos a los decisores públicos y privados. Para ello, este artículo se estructura como sigue. En primer lugar nos detendremos en el concepto de gobernanza como marco de este trabajo, mientras que las secciones subsiguientes se ocuparán del análisis de la gobernanza durante las primeras y últimas fases de la pandemia, finalizando con las implicaciones políticas, económicas y sociales que afectan al turismo rural.

\section{LA GOBERNANZA COMO MARCO DE ESTA INVESTIGACIÓN}

La estructuración territorial del Estado español se ha realizado fundamentalmente a través de un progresivo fortalecimiento de las Comunidades Autónomas, mientras que los gobiernos locales han adolecido de competencias fortalecedoras de su institucionalidad. De esta manera, en materia de turismo rural, la capacidad de actuación competencial y de despliegue de recursos descansa en las Comunidades Autónomas, si bien algunas Diputaciones Provinciales, como segundo nivel de gobierno local tienen capacidad para realizar actuaciones de fomento del turismo rural, pero la arquitectura es muy desigual en el conjunto del territorio español.

En este orden de cosas, de limitada codecisión en las políticas públicas estatales y regionales, la irrupción de la crisis desatada por el coronavirus ha hecho necesario el replanteamiento de las estrategias más cortoplacistas de los gobiernos locales. Con toda seguridad, la gestión de la crisis y de sus incertidumbres ha obligado a los gobiernos locales a concentrar sus energías políticas y de gestión para dar respuesta a las urgencias de la misma. Urgencias que se han presentado en los 8.125 municipios que existen en España, de los cuales 4.955 tienen menos de 1.000 habitantes según los últimos datos del INE (2019). 
El concepto de gobernanza ocupa un lugar central en el debate de la Ciencia Política entendida como "un proceso de coordinación de actores, de grupos sociales y de instituciones, cuya perspectiva es obtener resultados definidos y deliberados colectivamente" (Le Galès, 2010). La gobernanza se ocupa de cómo movilizar un conjunto de actores críticos (grupos de interés, asociaciones cívicas, ONGs, entre otros) para, mediante procesos de acción colectiva e interdependencias, crear nuevas formas de colaboración a partir del conflicto, de integración a partir de la fragmentación y de coherencia a partir de la inconsistencia. Y lo hace a partir de la búsqueda de consensos en los que están involucrados diversos actores públicos y privados en el empeño de definir objetivos comunes a partir de normas acordadas entre los miembros de la red, acordar la contribución de cada uno de ellos en la consecución de esos objetivos, no siempre concurrentes, y acordar una visión común acerca del futuro de su territorio. Sobre el concepto de gobernanza existe una extensa literatura científica que no es posible abordar en su totalidad, pero en la que se enfatizan aspectos como la actividad en red, la participación ciudadana, la gestión pública y el capital social (Conejero, 2005; Aguilar, 2007; Dredge y Pforr, 2008; Trunfio y Della Lucia, 2013, entre otros).

La gobernanza territorial es una extensión de la gobernanza multinivel pero que se focaliza en una demarcación territorial e interesada en las especificidades del territorio. En conjunto, la gobernanza genera activos intangibles, impulsa modificaciones en el modelo de organización económica, mejora en la calidad democrática local en el proceso de toma de decisiones y en tales como capital social y relacional. Por ello, es posible sostener que la competitividad de un determinado territorio no depende tanto de su capital territorial sino del modelo de gobernanza adoptado, es decir, que su ventaja comparativa viene determinada por la calidad de las redes de colaboración generadas desde abajo (down-top) y no de modelos definidos cuantitativamente desde arriba (top-down). En otros términos, la gobernanza local es una precondición para la generación de capital territorial en donde los gobiernos locales, como impulsores de interacciones y sinergias y como agentes públicos sujetos a procesos de rendición de cuentas son susceptibles de convertirse en actores relevantes en el contexto de la globalización. Su visibilidad, accesibilidad y proximidad con respecto a la ciudadanía refuerza los efectos sinérgicos.

De este presupuesto se sigue para la optimización de la acción pública en materia de desarrollo territorial, en los que el turismo rural juega y está abocado a tener un creciente protagonismo, son los gobiernos locales los mejor situados para generar procesos endógenos de desarrollo territorial integrado tanto en términos de eficacia como de democracia que son los gobiernos locales. Y a lo largo de este trabajo trataremos de poner de manifiesto que son los mejor situados, ya que muchas de las fuerzas que operan en la economía global no lo hacen a nivel local, lo cual hace relevante el concepto de gobernanza local.

Con todo ello, a medio y largo plazo y en el contexto de la crisis COVID-19 la gobernanza local se inscribe en un doble contexto. En primer lugar, en el contexto de recuperación nacional diseñado mediante la publicación de un real decreto a finales del año 2020. Este plan de recuperación nacional remitido a las instituciones de la Unión Europea contempla que las administraciones públicas sean impulsoras de la recuperación a nivel estatal. Naturalmente, esta acción impulsora o tractora se haría extensible a los gobiernos locales en cuyo contexto, las políticas de recuperación llevarían implícito un reforzamiento de la gobernanza local. Con todo, en la agenda política de la recuperación a la hora de escribir este texto los gobiernos locales no tienen ningún protagonismo en esta recuperación. Lo que sí es cierto es que los fondos europeos de recuperación que va a recibir el Estado español se va a constituir como el elemento capital en los procesos de gobernanza. En segundo lugar, 
en el contexto de la agenda 2030 se contemplan diversos ejes de actuación: económico, social, medioambiental y, ciertamente, uno transversal que es la gobernanza a la que se hace referencia para la gestión de los fondos y, con estas premisas, la gobernanza se considera un instrumento imprescindible para la implementación de los diferentes objetivos de la Agenda 2030.

En otros términos y desde el punto de vista de la gobernanza local esta Agenda 2030 sirve de referencia estratégica para la acción local al plantear un horizonte temporal de 10 años. Ello significa que los gobiernos locales están obligados a enfrentarse a los desafíos ya establecidos en la Agenda 2030 y a los futuros derivados de la pandemia. Lo cierto es que para afrontar los retos presentes y futuros España va a recibir no sólo los fondos sociales y regionales tradicionales de la UE, sino también los fondos de recuperación. En esta coyuntura y dado el sistema de gobernanza multinivel del Estado español, es probable que parte de estos recursos sean gestionados por las entidades locales.

\section{UN APUNTE METODOLÓGICO}

En este trabajo intentamos dar cuenta de un fenómeno que todavía está ocurriendo y cuya aproximación exige, a nuestro entender, una primera aproximación cualitativa. Así pues, examinaremos los planes de contingencia para documentar las acciones más efectivas que pueden servir de referencia para futuras situaciones similares. Para ello, nos centraremos en el turismo rural y en las acciones de emergencia habilitadas para hacer frente a la situación.

De esta forma, nuestro estudio se sustenta en una serie de entrevistas semiestructuradas a actores críticos que incluyen decisores públicos, expertos en turismo rural y empresarios y asociaciones empresariales de turismo rural. Se han llevado a cabo 20 entrevistas que incluyen a 4 expertos de la Federación Española de Municipios, 3 altos cargos ministeriales, 10 empresarios de turismo rural, 3 representantes de asociaciones empresariales. Los cargos públicos han sido incluidos porque se han visto involucrados en el diseño de las acciones y los empresarios y asociaciones de empresarios porque han sufrido los impactos $\mathrm{y}$ consecuencias de la pandemia y son conocedores de los impactos de las medidas adoptadas y de las acciones de cara al futuro. Se ha preguntado a los entrevistados y se ha indagado en el análisis documental acerca de los siguientes temas: en primer lugar en lo relativo a su percepción en los mecanismos de coordinación horizontal y vertical entre los actores críticos e instituciones competentes en materia de turismo rural, el liderazgo político, la cooperación intermunicipal, su capacidad de gestión estratégica y el papel de las Comunidades Autónomas y de las Diputaciones provinciales. En segundo lugar, acerca de la integración de las políticas y programas públicos, su grado de integración o fragmentación y su impacto sobre el turismo rural. En tercer lugar, acerca de la movilización de los actores críticos, los procesos de toma de decisiones y la rendición de cuentas. Debido a las restricciones perimetrales las entrevistas han sido realizadas telefónicamente y el acceso a la documentación se ha visto también limitado por las condiciones de movilidad impuestas por la pandemia.

Ello nos permitirá identificar los lineamientos generales de las estrategias, planes, programas, políticas públicas y acciones que se han adoptado para hacer frente a los impactos de la pandemia en los ámbitos de la salud, sociales y de la economía local, incluido el turismo rural e identificar las innovaciones en la gobernanza de emergencias en términos de liderazgo, cooperación y colaboración entre los actores críticos y la gestión de los datos, así como los cambios introducidos en las relaciones interadministrativas, en la gobernanza 
multinivel y en la cooperación intermunicipal para afrontar los impactos de la pandemia en el turismo rural.

Se ha llevado a cabo también un análisis de los documentos oficiales elaborados por la administración de la UE relacionados con el turismo rural, en tanto en cuanto sus fondos Leader que son canalizados a través de las Consejerías y con la colaboración de grupos de la sociedad civil local, y que se constituyen como un instrumento de dinamización de la economía rural $\mathrm{y}$, en particular del turismo rural como actividad innovadora local en cada territorio.

\section{LA GOBERNANZA LOCAL DURANTE LA PANDEMIA}

Según la encuesta de ocupación más reciente las pernoctaciones en alojamientos turísticos de carácter rural ha sido en el mes de Febrero de 2021 un 78,2\% menor a la misma ocupación que en el mismo mes del año 2020, aunque con desigual incidencia dependiendo de las Comunidades Autónomas y, entre estas por provincias. A fecha de este estudio no se conocen con exactitud las cifras de la incidencia por municipios, si bien se sabe que, en términos generales el turismo rural fue una opción preferida durante los meses de julio y agosto de 2020, aunque habrá que esperar a que se recopilen datos estadísticos fiables que muestren otros parámetros identificadores de la composición tanto de la demanda como de la oferta una vez se vuelva a una situación más parecida a la de pre-pandemia.

En conjunto, la gobernanza local durante la pandemia se ha sustentado fundamentalmente en la colaboración y cooperación interinstitucional y con la sociedad civil. Esta cooperación no ha sido pacífica en un sistema descentralizado como es el español, lo que ha significado la existencia de múltiples fricciones entre el gobierno central y los gobiernos regionales de las Comunidades Autónomas. En este contexto de cooperación/conflicto entre las autoridades centrales y regionales los gobiernos locales han jugado un papel ancilar. Así, han sido el gobierno central y los gobiernos regionales quienes a través de Conferencias de Presidentes han tomado las decisiones relativas a los confinamientos durante la proclamación y sucesivas extensiones del estado de alarma. En este contexto se han establecido planes de intervención en cada municipio que, en lo que se refiere a la actuación en materia de turismo rural se ha materializado fundamentalmente en la capacitación e información de protocolos de seguridad. En lo que se refiere a los confinamientos perimetrales, su incidencia ha sido disímil en función de los territorios. Así, en la Comunidad de Madrid, los alojamientos turísticos localizados en los pueblos de la Sierra Norte se han visto beneficiados por la afluencia de visitantes, sobre todo los fines de semana. Esto, sin duda, ha beneficiado a los alojamientos turísticos locales, pero por otra parte ha sido percibido como una amenaza por buena parte la población autóctona por miedo a los contagios. Aunque la fase de desescalada fue diseñada por el gobierno central sustentándose en los informes de los expertos, en la práctica la implantación de las diferentes fases de desescalada ha sido llevada a efecto por las Comunidades Autónomas, lo que ha significado la toma de decisiones no coincidentes en materia de permisibilidad a la movilidad que está en la base de la actividad turística. En este marco, la actividad de los establecimientos rurales, en las fases de desescalada ha estado, por tanto, determinada por las diferentes estrategias llevadas a efecto por las Comunidades Autónomas.

A este respecto, el hecho de que cada Comunidad Autónoma disponga de su propia normativa reguladora de la actividad turística rural ha significado que las medidas hayan adoptado un amplio espectro dependiendo del territorio. Por lo demás, a esta diversidad 
normativa ha de añadirse la existencia de diferentes tipologías de alojamientos rurales, pero, en este caso, las casas pequeñas con grupos limitados al alojamiento de seis personas o menos se han visto beneficiadas y, teniendo en cuenta que las casas rurales son la punta de lanza del turismo rural para el fomento del autoempleo femenino, se puede asegurar que en esta tipología de alojamientos se ha preservado el empleo.

En cualquier caso, los actores críticos entrevistados han puesto de manifiesto la inexistencia de coordinación horizontal y vertical entre los agentes e instituciones responsables en materia de turismo rural. La obligada ausencia de liderazgo político por parte de los alcaldes de las zonas rurales y la mínima capacidad de gestión estratégica y operativa han abocado al cierre de múltiples establecimientos de turismo rural. También se ha echado de menos un papel más activo de las Comunidades Autónomas en este sector, aunque las diputaciones provinciales hayan sido los actores más dinámicos y que han actuado como primer dique de contención a los problemas del sector no se han producido sinergias entre los propietarios y gestores de los establecimientos y de los actores públicos durante la pandemia. En este orden de cosas, si bien las diputaciones provinciales como gobiernos locales del segundo escalón han proporcionado al sector del turismo rural subvenciones a fondo perdido, no se han tomado medidas significativas en materia fiscal.

\section{LA GOBERNANZA POST-PANDEMIA: PROBLEMAS Y RETOS}

Con toda seguridad, en la post-pandemia se requiere una importante actividad de fomento por parte de los poderes públicos para reactivar la economía y en esta actividad los gobiernos locales están llamados a desempeñar un importante papel en lo que se refiere a la potenciación del turismo rural. Sin duda, en este contexto los fondos de recuperación de los que se ha dotado la Unión Europea para hacer frente a las consecuencias de la pandemia significan para España una oportunidad para la introducción de reformas estructurales que permitan la implantación de un modelo rural que haga frente a los retos del despoblamiento y sus consecuencias sociales, económicas y medioambientales con la vista puesta en la mejora de la cohesión social y territorial a partir de una búsqueda de reequilibrios entre el mundo urbano y rural para lo que se requieren importantes inversiones en este último. En todos estos aspectos la actividad turística en rural será receptora de ayuda en tanto en cuanto las Comunidades Autónomas y los gobiernos locales está previsto que se ocupen de gestionar un buen porcentaje de esos fondos. En concreto, de los más de 1,8 billones que la Unión Europea destinará a la recuperación de la Europa post-pandemia los gobiernos regionales y locales dispondrán de un monto aproximado de 35.000 millones de euros, parte de los cuales irán a financiar el Plan Nacional de Sostenibilidad Turística en Destinos cuya vigencia se desarrollará en los próximos tres años, de 2021 a 2024, y que se enmarca dentro de la Estrategia 2030 para Turismo sostenible. Tanto en la Estrategia 2030 como en el Plan Nacional de Sostenibilidad se da una especial relevancia a la modalidad del turismo rural en tanto en cuanto que es una actividad clave para abordar el despoblamiento, la conservación del patrimonio cultural y la sostenibilidad medioambiental. Y para la eficaz implementación de las acciones que se contemplen en estos planes es imprescindible la participación de los gobiernos locales, pues sólo ellos son los conocedores de las diversas singularidades de cada territorio. Por otra parte, las medidas que se implanten para la consecución de los objetivos tanto de la Estrategia como del Plan requieren de procesos de gobernanza en los que no sólo este presente la colaboración entre los diferentes niveles territoriales de gobierno y administración, sino también con la participación de los actores críticos que conforman el 
tejido social en cada territorio mediante el establecimiento de grupos de trabajo conformados por representantes de la sociedad civil local que sean capaces de generar el máximo de inteligencia territorial y a diferentes escalas. Ello sólo se conseguirá con una importante movilización de los actores críticos de cada territorio quienes en alianza con las diferentes administraciones públicas, con el tercer sector, con el sector privado, con el tejido asociativo rural y con otras instituciones (por ejemplo, de investigación) sean capaces de la creación de redes de acción en cada territorio para el fomento de su economía $\mathrm{y}$, en particular, del desarrollo del turismo rural.

Correspondería en todo caso a las Comunidades Autónomas y, en lo que se refiere a los pequeños municipios rurales, a su Diputación Provincial el desarrollo de programas innovadores, puesto que los municipios rurales no tienen capacidad para ello. En algunas Comunidades Autónomas son también las comarcas, por su naturaleza inclusiva, las que están llamadas a generar estructuras compartidas que dinamicen la actividad turística en los municipios rurales. En este contexto de gobernanza multinivel los gobiernos locales de los pequeños municipios tendrían que ser dotados de capacidad de gestión, de manera que la gestión de los recursos para hacer frente a las consecuencias de la pandemia se puede ver como una oportunidad para los gobiernos locales de zonas rurales y transformar su capacidad de actuación. Así, es imprescindible que se potencien las obras de infraestructura que permitan y faciliten un mejor acceso a las localidades turísticas rurales, así como al apoyo de las fiestas locales y otras actividades culturales. En ello seguirá siendo imprescindible el papel tanto de las Diputaciones Provinciales como de los Cabildos Insulares, especialmente de estos últimos, pues en los municipios de la España insular una gran parte de su población depende de los ingresos derivados de la actividad turística.

Desde luego, con la vuelta a la normalidad, es fundamental hacer un balance de la situación de los establecimientos turísticos rurales de manera que se pueda conocer el impacto de la pandemia en cada uno de ellos al objeto de adoptar las medidas necesarias para su recuperación. Ni que decir tiene que las administraciones públicas, incluidos los gobiernos locales, tendrán que elaborar esos planes de recuperación a partir de las sugerencias que puedan hacer los propietarios y responsables de los establecimientos turísticos rurales.

A este respecto, otro de los retos de futuro en el ámbito del turismo rural y en el que los gobiernos locales deben participar es la a mejora de la conectividad que permita, entre otros, la extensión de la fibra para optimizar las opciones de comercio electrónico y la gestión y administración, así como los dispositivos para la seguridad en casos de emergencia. En este $\mathrm{y}$ otros aspectos son los gobiernos locales los que tienen que dotarse de capacidades propositivas para elevar propuestas a otras administraciones públicas. De la misma forma, cualquier decisión acerca de las políticas y programas de fomento del desarrollo local tienen que estar sustentados en la situación actual de las infraestructuras y equipamientos en los municipios. En este sentido, la actual encuesta de infraestructuras locales no detalla fielmente la situación en tanto en cuanto se trata más de una recopilación a modo de inventario y, a partir de ella no se pueden deducir las necesidades de las personas y empresarios que viven la realidad del mundo rural. Aquí, son también los gobiernos locales quienes tienen que adoptar un nuevo protagonismo para trasladar a las administraciones públicas la realidad de cada lugar de manera que en la normativa elaborada tanto por la administración central como por las administraciones autonómicas y que afecte a los gobiernos locales se tenga en cuenta la perspectiva de estos últimos. Además, en esta normativa se hace preciso introducir la perspectiva demográfica a modo de discriminación 
positiva y de la misma forma que en distintas normativas se introduce la perspectiva de género.

En cualquier caso, los ámbitos que han sido afectados por la pandemia son múltiples y es necesario coordinar y concertar las políticas y programas que los diferentes niveles de gobiernos territoriales aplican a los municipios rurales. En lo que se refiere a estos programas se requiere una acción integrada que tenga en su foco la ayuda a los empresarios autónomos que constituyen el grueso de la oferta turística rural con una flexibilización de las normas fiscales y el asesoramiento on-line a emprendedores locales.

Básicamente, si algo ha puesto de manifiesto la pandemia es que las administraciones públicas no disponen de la información suficiente para la adopción de decisiones con carácter de urgencia. Fijados en el territorio los gobiernos locales, si se les dota de recursos suficientes, son los que mejor están capacitados para generar información relativa a las dinámicas sociales y económicas de su entorno y hacerla fluir no sólo a otras administraciones públicas, sino a otros actores de la sociedad civil, facilitando así la atracción de inversiones para desarrollar las potencialidades locales. Quizás uno de los problemas a los que se enfrenta el mundo rural es la ausencia de emprendedores en los pueblos. Para que el emprendimiento se posible es preciso incorporar a los gobiernos locales como agentes que desarrollen un papel activo para motivar a sus vecinos en la realización de actividades encaminadas al desarrollo endógeno local.

En conjunto, el Plan de Recuperación, Transformación y Resiliencia elaborado con la finalidad de reactivar la economía ante las consecuencias derivadas del coronavirus se plantea como un marco en el que los gobiernos locales pueden presentar proyectos de carácter turístico. En esta línea, las convocatorias del Ministerio para la Transición Ecológica y el Reto Demográfico, concretadas en las Manifestaciones de Interés Reto Demográfico intentan recoger aquellas iniciativas presentadas por los Ayuntamientos para la reactivación de su territorio. Entre las áreas de actuación se contemplan tanto la recuperación del patrimonio y la promoción de proyectos culturales como el fomento de los destinos turísticos en el entorno rural. Para permitir diseñar estas propuestas, los gobiernos locales de carácter rural que no disponen de medios ni capacidades técnicas para su elaboración deberían contar con el apoyo y colaboración de las Diputaciones Provinciales o, en su caso, de las Comunidades Autónomas, quienes no pueden permitirse el lujo de ignorar la capacidad que tiene el turismo rural para contribuir al desarrollo sostenible endógeno del territorio y lograr, así, mayores cotas de cohesión social y territorial. Ciertamente, las diputaciones provinciales son importantes, pero en muchos casos, los problemas habría que abordarlos comarcalmente. Así, las carencias de la gestión de los pequeños municipios en relación con la gestión de los fondos europeos habrá de ser abordada mediante la asistencia para cubrir sus déficits en la capacidad de gestión. Por lo mismo, la agilización administrativa para la canalización de los fondos europeos se constituye en un elemento capital para la introducción de innovaciones en la gobernanza organizativa.

Desde luego, la crisis se presenta como una oportunidad para modificar la legislación y mejorar los procesos de gobernanza multinivel y de toma de decisiones otorgando a los gobiernos locales, como conocedores del territorio, un papel más relevante en la gestión de crisis globales cuyos impactos se manifiestan localmente.

\section{CONCLUSIONES Y LIMITACIONES DE ESTE ESTUDIO}


Con toda seguridad los efectos tanto directos como indirectos de la pandemia están aún por manifestarse. De hecho, es una crisis que no va a solucionarse a corto plazo, aunque, también es posible que los valores del turista/visitante estén cambiando, lo que representa una oportunidad para el turismo rural. En este sentido, este trabajo contribuye al debate acerca de las oportunidades que ofrece la crisis de la COVID-19 para el desarrollo del turismo rural y el papel que deben jugar los gobiernos locales bajo la premisa de que las crisis pueden ser aprovechadas porque ofrecen oportunidades de mejora o ventanas de oportunidad acelerando cambios que ya se estaban produciendo. Sin duda, la epidemia de COVID-19 ha creado una oportunidad para el desarrollo del turismo rural ya que es probable que la crisis fomente el deseo de realizar actividades al aire libre de los turistas de origen nacional, de la misma forma que el incremento del teletrabajo contribuya a la búsqueda de la naturaleza que ofrecen los entornos rurales. Ciertamente, los atractivos naturales, gastronómicos y locales ofrecen una situación favorable para difundir el turismo rural como destino seguro, no masificado, atractivo y sostenible y una oportunidad para reinventar la oferta con el diseño de estrategias innovadoras en el marco de desarrollo local y de los lineamientos contemplados en la Agenda 2030. En este contexto se podría afirmar la necesidad de que el turismo en general debe evolucionar hacia un modelo más sostenible que ponga en valor el territorio y su patrimonio, gastronomía y biodiversidad. Sabido es que Francia es el primer destino turístico del mundo y su oferta descansa en la venta de sol y playa, sino más bien en la oferta del interior del país, sus pueblos, localidades y su patrimonio antropológico y cultural. En otros términos, el turismo rural ofrece espacios de sostenibilidad, de diversificación, donde el turista sienta un valor añadido en su estancia.

Por otra parte, el turismo rural es una actividad que contribuye a la cohesión social y territorial no sólo poniendo freno a la despoblación, sino a la conservación del territorio mediante la promoción y protección del patrimonio y de los recursos naturales. Como motor económico contribuye a la diversificación y reactivación de otros sectores, industriales y de servicios imbricados con el turismo y es un motor de innovación. Si algo ha puesto de manifiesto la crisis de la COVID-19 es la necesidad de mejorar y fortalecer la coordinación vertical y horizontal de los diversos poderes territoriales y de estos con la sociedad civil.

En este contexto, y dado el importante papel que el turismo rural tiene en el desarrollo de los municipios, los gobiernos locales están abocados a diseñar nuevas estrategias concernientes al turismo rural que contribuyan a la mejora de las estructuras y equipamientos, con líneas de financiación disponibles y predecibles, con apoyo a las pequeñas empresas o al autoempleo fomentando la cooperación entre empresas, la cooperación intermunicipal e, incluso regional, creando imágenes atractivas para los territorios y fomentar prácticas innovadoras. En este orden de cosas cobran una capital importancia los gobiernos locales como facilitadores de un clima en el que se desarrollen capacidades de emprendimiento innovador que conduzcan a la creación de nuevos negocios turísticos. Aunque en España y en materia de turismo rural son las Comunidades Autónomas las que detentan la mayor capacidad para el diseño de políticas públicas con impacto en el turismo rural, los municipios, en razón de la cercanía, están en una posición privilegiada para apoyar a los emprendedores individuales, convirtiéndose así en los protagonistas de la colaboración público-privada mediante acciones de gobernanza que pongan en valor una visión colectiva del municipio como "comunidad local".

Sin duda, en España, un país cuyos ingresos derivados del turismo están por encima del $10 \%$ del PIB, los escenarios más catastróficos del declive del turismo provocados por la pandemia afectan principalmente a los destinos urbanos centrados y enfocados en el turismo extranjero. Por el contrario, los destinos rurales suponen una alternativa. Algunos de ellos, 
como ya se ha indicado, han mostrado un aumento del turismo nacional en la temporada de verano de 2020 en comparación con la de 2019. Sin embargo, esta nueva orientación requiere invertir en infraestructuras, marketing y cooperación interadministrativa. En este orden de cosas, las administraciones públicas desempeñan un papel importante tanto en la lucha contra la COVID-19 como en la recuperación de la economía, que incluye la industria del turismo. De esta forma hay que hacer énfasis en la necesidad de reforzar los procesos de gobernanza: colaboración, intercambio de información y coordinación entre los diferentes niveles de gobierno con un reforzamiento de las capacidades institucionales del nivel local, de las diputaciones provinciales y de los cabildos insulares para la elaboración de programas sobre el patrimonio cultural y la biodiversidad de manera que se conformen sistemas de colaboración con arquitecturas variables que incluyan la sistematización análisis y gobernanza de datos. En definitiva, la potenciación de los gobiernos locales en materia de promoción de la actividad económica y del empleo en sus territorios es clave para la recuperación de la España rural en tanto en cuanto los pequeños municipios son idóneos para el desarrollo de la economía creativa por lo que se hace preciso convertir a los gobiernos locales en gestores de su territorio.

Con todo, este estudio presenta algunas limitaciones. La primera es que la pandemia es un fenómeno que todavía está ocurriendo y no es posible obtener un conocimiento del panorama en su conjunto, con datos e información aún no disponibles. Ciertamente, se hacen precisos estudios ulteriores de carácter interdisciplinario que sirvan de orientación para hacer frente a crisis similares en el futuro y que permita a los responsables de la toma de decisiones la elaboración de estrategias y la planificación para reducir los impactos. A este respecto, la naturaleza interrelacionada de la industria turística requiere que los responsables de las políticas públicas consideren el sector desde una perspectiva fundamentalmente holística, por lo que no se puede simplemente diseñar acciones focalizadas única y exclusivamente en la ayuda, por ejemplo, a los establecimientos hoteleros y esperar que con ello se solucionen los problemas del turismo rural.

\section{BIBLIOGRAFÍA}

Aguilar, L.F. (2007) Gobernanza y Gestión Pública. Fondo de Cultura Económica, México.

Bouckaert, G; Van Hecke. S; Galli, D. et. al. (2020) European coronationalism? A hot spot governing a pandemic crisis. Public Administration Review, 80 (5), 765-773.

Conejero, E. (2005) Globalización, gobernanza local y democracia participativa, Cuadernos Constitucionales de la Cátedra Fadrique Furió Cerriol, 52-53, 13-31.

Dredge, D. y Pforr, C. (2008) "Policy Networks and Tourism Governance". En Cooper, C.; Scott, N y Baggio, R. (Coord.) Network Analysis and Tourism: From Theory to Practice, Channelview, doi: 10.21832/9781845410896-008.

Foris, D. (2014). Study regarding a new dimension of tourism phenomenon: the politicaladministrative dimension, Social and Behavioural Sciences, 143, 1078-1082.

Gössling, S.; Scott, D., Hall, C.M. ( ). Pandemics, tourism and global change: a rapid assessment of COVID-19. Journal of Sustainable Tourism

Instituto Nacional de Estadística. https://www.ine.es/dyngs/INEbase/es/operacion.htm?c=Estadistica C\&cid=1254736176963\&me nu=ultiDatos\&idp=1254735576863. Consultado el 15.4.2021.

Jiang, Y., \& Ritchie, B. W. (2017). Disaster collaboration in tourism: Motives, impediments and success factors. Journal of Hospitality and Tourism Management, 31, 70-82. https://doi.org/10.1016/i.jhtm.2016.09.004 
Kumar, Ch. (2020), Corona(COVID-19) and Tourism: More Opportunities In The Aftermath of The Lockdown, Academia.edu, Nota de investigación. Consultada el 26 de Abril de 2020.

Le Gales P. (2010). Gouvernance, En : Boussaguet L. et al., Dictionnaire des politiques publiques, Presses de Science Po, 299-308.

Martínez, J.M. (2019). An analysis of the stability of rural tourism as a desired condition for sustainable tourism. Journal of Business Research, 100, 165-74.

Novelli, M., Gussing Burgess, L., Jones, A., \& Ritchie, B. W. (2018). 'No Ebola... still doomed' - The Ebola-induced tourism crisis. Annals of Tourism Research, 70, 76-87. https://doi.org/10.1016/j.annals.2018.03.006

Panik, E. (2015). Rural tourism governance: determinants of policy-makers' support for tourism development. Tourism Planning and Development

Petrovic, M.D., Vujko, A., Gajic, T.,Vukovic, D., Radiovanovic, J.; Jovanovoc, J.; Vukovic, N., (2018). Tourism as an approach to sustainable rural developmen in post-socialist countries. A comparative study of Serbia and Slovenia. Sustainability, 10, 54.

Scott, N.; Laws, E y Prideaux, B (2008). Tourism Crises and Marketing Recovery Strategies',Journal ofTravel \& Tourism Marketing,23:2,1. DOI: http://dx.doi.org/10.1300/J073v23n02

Sharpley, R. y Roberts, L., (2004) Rural tourism-10 years on. International Journal of Tourism Research, 6, 119-24.

Sims, R. (2009). Food, place and authenticity: Local food and the sustainable tourism experience. Journal of Sustainable Tourism, 17, 321-36

Tirado, J.G. y Hernández, M. ( 2018). Promoting tourism through the EU LEADER programme: understanding Local Action Group. European Planning Studies

Trunfio, M. y Della Lucia, M. (2013). Social Capital and Governance for Sustainable Rural Development Studies in Agricultural Economics, 115 (2), 104-110. DOI: 10.7896/j.1220.

\section{Breve currículo}

\section{Ángel Iglesias Alonso}

Ángel Iglesias Alonso es Profesor Titular de Ciencia Política y de la Administración de la Universidad Rey Juan Carlos. Ha participado en varios proyectos de investigación de $\mathrm{I}+\mathrm{d}+\mathrm{i}$ competitivos que tienen como objeto de estudio los gobiernos locales, de carácter nacional e internacional (H2020), bien como investigador o investigador principal y ha publicado los resultados en varias decenas de artículos científicos en revistas nacionales e internacionales indexadas y en libros y capítulos de libros de los primeros cuartiles de la IPA. 